\title{
New Classes of Odd Graceful Graphs
}

\author{
M. E. Abdel-Aal \\ Department of Mathematics, Faculty of Science, \\ Benha University, Benha 13518, Egypt \\ mohamed_el77@yahoo.com
}

\begin{abstract}
In this paper, we introduce the notions of m-shadow graphs and $n$-splitting graphs, $m \geq 2, n \geq 1$. We prove that, the m-shadow graphs for paths, complete bipartite graphs and symmetric product between paths and null graphs are odd graceful. In addition, we show that, the m-splitting graphs for paths, stars and symmetric product between paths and null graphs are odd graceful. Finally, we present some examples to illustrate the proposed theories.
\end{abstract}

\section{KEYWORDS}

Odd graceful, m-shadow graph, m-splitting graph, Symmetric product.

\section{INTRODUCTION}

Graph labeling have often been motivated by practical problems is one of fascinating areas of research. A systematic study of various applications of graph labeling is carried out in Bloom and Golomb [1]. Labeled graph plays vital role to determine optimal circuit layouts for computers and for the representation of compressed data structure.

The study of graceful graphs and graceful labelling methods was introduced by Rosa [2]. Rosa defined a $\beta$-valuation of a graph $G$ with $q$ edges as an injection from the vertices of $\mathrm{G}$ to the set $\{0,1,2, \ldots, q\}$ such that when each edge xy is assigned the label $|f(x)-f(y)|$, the resulting edge labels are distinct. $\beta$-Valuations are the functions that produce graceful labellings. However, the term graceful labelling was not used until Golomb studied such labellings several years later [3]. The notation of graceful labelling was introduced as a tool for decomposing the complete graph into isomorphic subgraphs.

We begin with simple, finite, connected and undirected graph $G=(V, E)$ with $p$ vertices and $q$ edges. For all other standard terminology and notions we follow Harary[5].

Gnanajothi [6] defined a graph $G$ with $q$ edges to be odd graceful if there is an injection $f$ from $V(G)$ to $\{0,1,2, \ldots, 2 q-1\}$ such that, when each edge $x y$ is assigned the label $|f(x)-f(y)|$. Seoud and Abdel-Aal [7] determine all connected odd graceful graphs of order at most 6 and they proved that if $G$ is odd graceful, then $G \cup K_{m, n}$ is odd graceful for all $m, n \geq 1$. In addition, they

DOI : 10.5121/jgraphoc.2013.5201 
International journal on applications of graph theory in wireless ad hoc networks and sensor networks (GRAPH-HOC) Vol.5, No.2, June 2013

proved that many families of graphs such as splitting of complete bipartite graph, Cartesian product of paths, symmetric product for paths with null graph, conjunction of paths and conjunction of paths with stars are odd graceful.

We know that, the shadow graph $D_{2}(G)$ of a connected graph $G$ is constructed by taking two copies of $G$ say $G^{\prime}$ and $G^{\prime \prime}$. Join each vertex $u$ in $G^{\prime}$ to the neighbors of the corresponding vertex $\mathrm{v}$ in $G^{\prime \prime}$. Also we know that, the splitting graph $G$ is obtained by adding to each vertex $v$ a new vertex $v^{\prime}$ such that $v^{\prime}$ is adjacent to every vertex which is adjacent to $v$ in $G$. The resultant graph is denoted by $\operatorname{Spl}(G)$.

Vaidya and Lekha [8] proved that the shadow graphs of the path $P_{n}$ and the star $K_{l, n}$ are odd graceful graphs. Further they proved in [9] that the splitting graphs of the star $K_{l, n}$ admit odd graceful labeling. Moreover, Sekar [10] has proved that the splitting graph of path is odd graceful graph. Also, Seoud and Abdel-Aal [7] proved that $\operatorname{Spl}\left(K_{n, m}\right), \operatorname{Spl}\left(P_{n} \oplus \overline{K_{2}}\right)$.

In this paper, we introduce an extension for shadow graphs and splitting graphs. Namely, for any integers $m \geq 1$, the $m$-shadow graph denoted by $D_{m}(G)$ and the $m$-splitting graph denoted by $\operatorname{Spl}_{m}(G)$ which are defined as follows:

Definition 1.1. The m-shadow graph $D_{m}(G)$ of a connected graph $G$ is constructed by taking $m$ copies of $G$, say $G_{1}, G_{2}, G_{3}, \ldots, G_{m}$, then join each vertex $u$ in $G_{i}$ to the neighbors of the corresponding vertex $v$ in $G_{j}, 1 \leq i, j \leq m$.

Definition 1.2. The $m$ - splitting graph $\operatorname{Spl}_{m}(G)$ of a graph $G$ is obtained by adding to each vertex $v$ of $G$ new $m$ vertices, say $v^{1}, v^{2}, v^{3}, \ldots, v^{m}$, such that $v^{i}, 1 \leq i \leq m$ is adjacent to every vertex that is adjacent to $v$ in $G$.

By definitions, the 2-shadow graph is the known shadow graph $D_{2}(G)$ and the 1 - splitting graph is the known splitting graph.

In our study, we generalize some results on splitting and shadow graphs by showing that, the graphs $D_{m}\left(P_{n}\right), D_{m}\left(P_{n} \oplus \overline{K_{2}}\right)$, and $D_{m}\left(K_{r, s}\right)$ for each $m, n, r, s \geq 1$ are odd graceful. Moreover, we also show that the following graphs $\operatorname{Spl}_{m}\left(P_{n}\right), \operatorname{Spl}_{m}\left(K_{l, n}\right), \operatorname{Spl}_{m}\left(P_{n} \oplus \overline{K_{2}}\right)$ are odd graceful. 
International journal on applications of graph theory in wireless ad hoc networks and sensor networks (GRAPH-HOC) Vol.5, No.2, June 2013

\section{MAIN RESULTS}

Theorem 2.1.

$D_{m}\left(P_{n}\right)$ is an odd graceful graph for all $m, n \geq 2$.

Proof. Consider $m$-copies of $P_{n}$. Let $u_{1}^{j}, u_{2}^{j}, u_{3}^{j}, \ldots, u_{n}^{j}$ be the vertices of the $\mathrm{j}^{\text {th }}$-copy of $P_{n}$, $1 \leq j \leq m$. Let $G$ be the graph $D_{m}\left(P_{n}\right)$, then $|V(G)|=m n$ and $q=|E(G)|=m^{2}(n-1)$. We define $f: V(G) \rightarrow\left\{0,1,2, \ldots, 2 m^{2}(n-1)-1\right\}$ as follows:

$$
f\left(u_{i}^{j}\right)=\left\{\begin{array}{lll}
2 q-1-m^{2}(i-1)-2 m(j-1) & i=1,3,5, \ldots, n \text { or } n-1, & 1 \leq j \leq m, \\
m^{2}(i-2)+2(j-1) & i=2,4,6, \ldots, n-1 \text { or } n, & 1 \leq j \leq m .
\end{array}\right.
$$

The above defined function $f$ provides odd graceful labeling for $D_{m}\left(P_{n}\right)$. Hence $D_{m}\left(P_{n}\right)$ is an odd graceful graph for each $m, n \geq 1$.

Example 2.2. An odd graceful labeling of the graph $D_{4}\left(P_{6}\right)$ is shown in Figure 1.

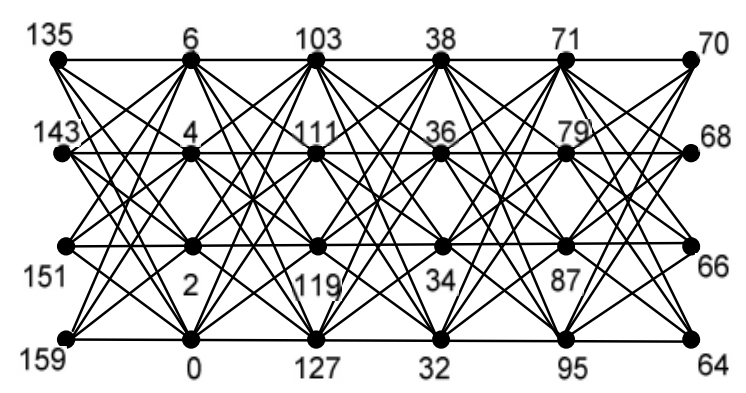

Figure 1: The graph $\mathrm{D}_{4}\left(\mathrm{P}_{6}\right)$ with its odd graceful labeling.

Theorem 2.3. $\mathrm{D}_{\mathrm{m}}\left(\mathrm{K}_{\mathrm{r}, \mathrm{s}}\right)$ is an odd graceful graph for all $\mathrm{m}, \mathrm{r}, \mathrm{s} \geq 1$.

Proof. Consider m-copies of $\mathrm{K}_{\mathrm{r}, \mathrm{s}}$. Let $u_{1}^{j}, u_{2}^{j}, u_{3}^{j}, \ldots, u_{r}^{j}$ and $v_{1}^{j}, v_{2}^{j}, v_{3}^{j}, \ldots, v_{s}^{j}$ be the vertices of the $\mathrm{j}^{\text {th }}$-copy of $\mathrm{K}_{\mathrm{r}, \mathrm{s}}, 1 \leq j \leq m$. Let $\mathrm{G}$ be the graph $\mathrm{D}_{\mathrm{m}}\left(\mathrm{K}_{\mathrm{r}, \mathrm{s}}\right)$, then $|\mathrm{V}(\mathrm{G})|=\mathrm{m}(\mathrm{r}+\mathrm{s})$ and $\mathrm{q}=|\mathrm{E}(\mathrm{G})|$ $=\mathrm{m}^{2}$ rs. We define

$$
\mathrm{f}: \mathrm{V}(\mathrm{G}) \rightarrow\left\{0,1,2, \ldots, 2 \mathrm{~m}^{2} \mathrm{rs}-1\right\}
$$

as follows:

$$
\begin{array}{ll}
f\left(u_{i}^{j}\right)=2(i-1)+2 r(j-1), & 1 \leq i \leq r, 1 \leq j \leq m . \\
f\left(v_{i}^{j}\right)=2 q-1-2 m r(i-1)-2 m r s(j-1), & 1 \leq i \leq s, 1 \leq j \leq m .
\end{array}
$$


International journal on applications of graph theory in wireless ad hoc networks and sensor networks (GRAPH-HOC) Vol.5, No.2, June 2013

Above defined labeling pattern exhausts all possibilities and the graph under consideration admits odd graceful labeling. Hence $\mathrm{D}_{\mathrm{m}}\left(\mathrm{K}_{\mathrm{r}, \mathrm{s}}\right)$ is an odd graceful graph for each $\mathrm{m}, \mathrm{r}, \mathrm{s} \geq 1$.

Example 2.4. An odd graceful labeling of the graph $\mathrm{D}_{3}\left(\mathrm{~K}_{3,4}\right)$ is shown in Figure 2 .

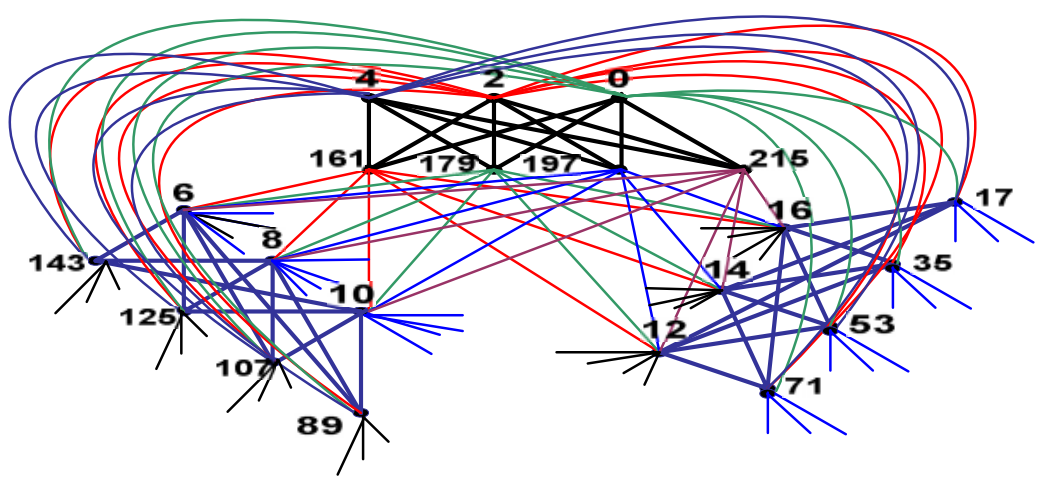

Remark 2.5.

Figure 2: The graph $D_{3}\left(K_{3,4}\right)$ with its odd graceful labeling.

In Theorem 2.1, if we take $m=2$ we obtain the known shadow path also, when we take $m=2, r$ $=1$ in Theorem 2.3 we obtain the known shadow star. These special cases of our results are coincided with Vaidya's results in [8, theorems $2.6,2.4]$; respectively.

Let $G_{1}$ and $G_{2}$ be two disjoint graphs. The symmetric product $\left(G_{1} \oplus G_{2}\right)$ of $G_{1}$ and $G_{2}$ is the graph having vertex set $V\left(G_{1}\right) \times V\left(G_{2}\right)$ and edge $\operatorname{set}\left\{\left(u_{1}, v_{l}\right)\left(u_{2}, v_{2}\right): u_{1} u_{2} \in E\left(G_{1}\right)\right.$ or $v_{1} v_{2} \in E\left(G_{2}\right)$ but not both\}[4].

In [11] Seoud and Elsakhawi shown that $P_{2} \oplus \overline{K_{2}}$ is arbitrary graceful, and in [7] Seoud and Abdel-Aal proved that the graphs $P_{n} \oplus \overline{K_{m}}, m, n \geq 2$ are odd graceful. The next theorem shows that the $m$-shadow of $\left(P_{n} \oplus \overline{K_{2}}\right)$ for each $m, n \geq 2$ is odd graceful.

Theorem 2.6.

The graph $\mathrm{D}_{\mathrm{m}}\left(\mathrm{P}_{\mathrm{n}} \oplus \overline{K_{2}}\right), \mathrm{m}, \mathrm{n} \geq 2$ is odd graceful. 
International journal on applications of graph theory in wireless ad hoc networks and sensor networks (GRAPH-HOC) Vol.5, No.2, June 2013

\section{Proof.}

Let $u_{1}^{1}, u_{2}^{1}, u_{3}^{1}, \ldots, u_{n}^{1}, v_{1}^{1}, v_{2}^{1}, v_{3}^{1}, \ldots, v_{n}^{1}$ be the vertices of $\mathrm{P}_{\mathrm{n}} \oplus \overline{K_{2}}$ and suppose $u_{1}^{j}, u_{2}^{j}, u_{3}^{j}, \ldots, u_{n}^{j}$, $v_{1}^{j}, v_{2}^{j}, v_{3}^{j}, \ldots, v_{n}^{j} \quad$ be the $\mathrm{j}^{\text {th }} \quad$-copy of $\mathrm{P}_{\mathrm{n}} \oplus \overline{K_{2}}, 1 \leq j \leq m$. Then the graph $\mathrm{G}=\mathrm{D}_{\mathrm{m}}\left(\mathrm{P}_{\mathrm{n}} \oplus \overline{K_{2}}\right)$ can be described as indicated in Figure 3.

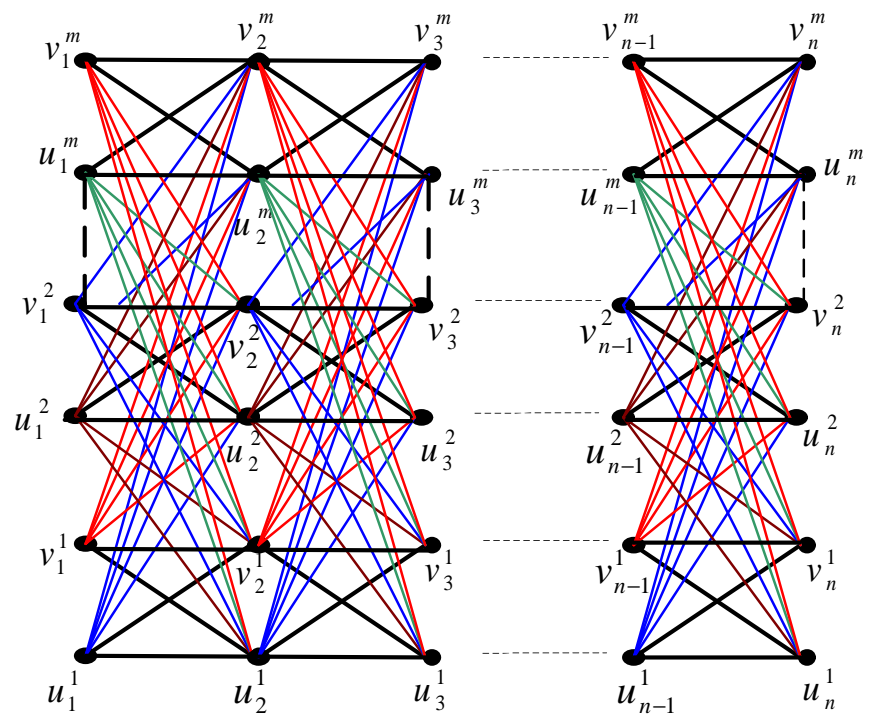

Figure 3

Then the number of edges of the graph $G$ is $4 m^{2}(n-1)$. We define:

$f: V(G) \rightarrow\left\{0,1,2, \ldots, 8 m^{2}(n-1)-1\right\}$

as follows:

$$
\begin{gathered}
f\left(u_{i}^{j}\right)= \begin{cases}(2 q-1)-4 m^{2}(i-1)-8 m(j-1), & i=1,3,5, \ldots n, j=1,2, \ldots, m \\
4 m^{2}(i-2)+4(j-1), & i=2,4,6, \ldots n, j=1,2, \ldots, m .\end{cases} \\
f\left(v_{i}^{j}\right)= \begin{cases}2\left[q-2 m^{2}(i-1)-4 m j+2 m\right]-1, & i=1,3,5, \ldots n, j=1,2, \ldots, m \\
4 m^{2}(j-2)+4 j-2, & i=2,4,6, \ldots n, j=1,2, \ldots, m .\end{cases}
\end{gathered}
$$

In accordance with the above labeling pattern the graph under consideration admits odd graceful labeling. Hence $D_{m}\left(P_{n} \oplus \overline{K_{2}}\right)$ is an odd graceful graph for each $m, n \geq 1$. 
International journal on applications of graph theory in wireless ad hoc networks and sensor networks (GRAPH-HOC) Vol.5, No.2, June 2013

Example 2.7. An odd graceful labeling of the graph $D_{3}\left(P_{4} \oplus \overline{K_{2}}\right)$ is shown in Figure 4.

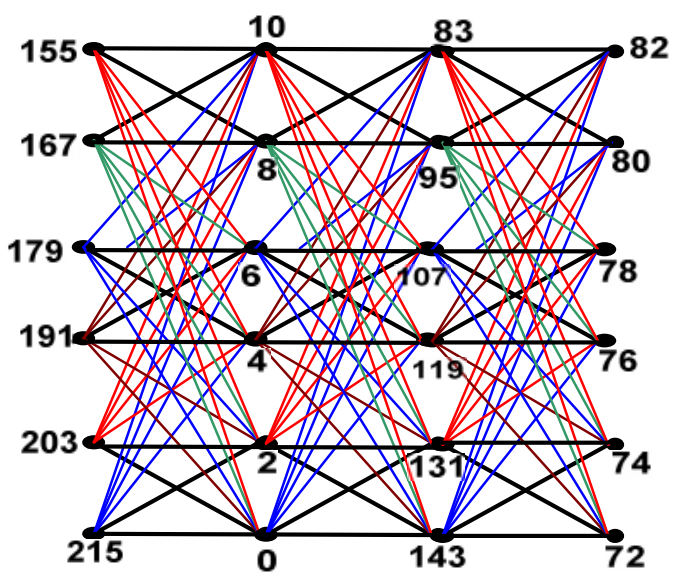

Figure 4: The graph $\mathrm{D}_{3}\left(\mathrm{P}_{4} \oplus \overline{K_{2}}\right)$ with its odd graceful labeling.

Theorem 2.8.

The graph $\mathrm{D}_{2}\left(\mathrm{P}_{\mathrm{n}} \times \mathrm{P}_{2}\right), \mathrm{n} \geq 2$ is odd graceful.

Proof. Let $u_{1}^{1}, u_{2}^{1}, u_{3}^{1}, \ldots, u_{n}^{1}, v_{1}^{1}, v_{2}^{1}, v_{3}^{1}, \ldots, v_{n}^{1}$ be the vertices of $\mathrm{P}_{\mathrm{n}} \times \mathrm{P}_{2}$ and suppose $u_{1}^{2}, u_{2}^{2}, u_{3}^{2}, \ldots, u_{n}^{2}, v_{1}^{2}, v_{2}^{2}, v_{3}^{2}, \ldots, v_{n}^{2}$, be the second copy of $\mathrm{P}_{\mathrm{n}} \times \mathrm{P}_{2}$. The graph $\mathrm{G}=\mathrm{D}_{2}\left(\mathrm{P}_{\mathrm{n}} \times \mathrm{P}_{2}\right)$ is described as indicated in Figure 5 .

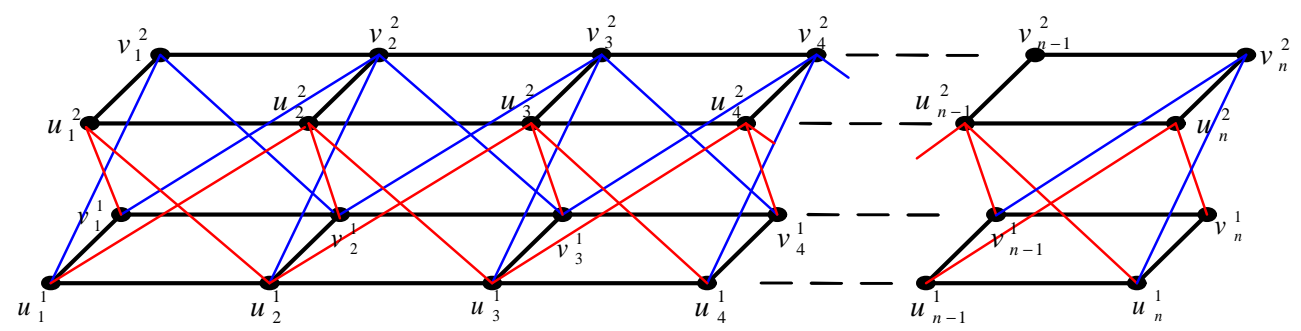

Clearly, the number of edges of the graph $G$ is $12 n-8$. We define:

$$
f: V(G) \rightarrow\{0,1,2, \ldots, 24 n-17\}
$$

as follows:

$$
f\left(u_{i}^{j}\right)= \begin{cases}2[q-6(i-1)-j]+1, & i=1,3,5, \ldots n, j=1,2, \\ 4(3 i+j-2), & i=2,4,6, \ldots n, j=1,2 .\end{cases}
$$


International journal on applications of graph theory in wireless ad hoc networks and sensor networks (GRAPH-HOC) Vol.5, No.2, June 2013

$$
f\left(v_{i}^{j}\right)= \begin{cases}4(3 i-j-1), & i=1,3,5, \ldots n \quad, j=1,2, \\ 2(q-6 i+j)+3, & i=2,4,6, \ldots n, j=1,2 .\end{cases}
$$

In view of the above defined labeling pattern the graph under consideration admits odd graceful labeling. Hence $D_{2}\left(P_{n} \times P_{2}\right)$ is an odd graceful graph for each $n \geq 2$.

Example 2.9. An odd graceful labeling of the graph $D_{2}\left(P_{n} \times P_{2}\right)$ is shown in Figure 6 .

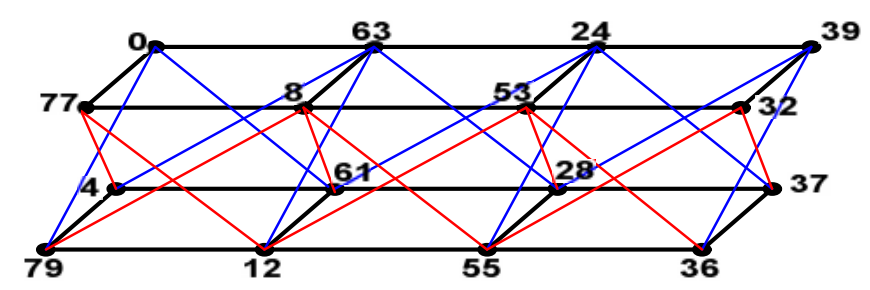

Figure 6: The graph $D_{2}\left(P_{n} \times P_{2}\right)$ with its odd graceful labeling.

\section{THE M-SPLITTING GRAPHS}

Theorem 3.1. The graph $\operatorname{Spl}_{m}\left(P_{n}\right)$ for each $m, n \geq 2$ is odd graceful.

Proof. Let $u_{1}^{0}, u_{2}^{0}, u_{3}^{0}, \ldots, u_{n}^{0}$ be the vertices of $P_{n}$ and suppose $u_{1}^{j}, u_{2}^{j}, u_{3}^{j}, \ldots, u_{n}^{j}, 1 \leq j \leq m$ be the $\mathrm{j}^{\text {th }}$ vertices corresponding to $u_{1}^{0}, u_{2}^{0}, u_{3}^{0}, \ldots, u_{n}^{0}$, which are added to obtain $\operatorname{Spl}_{m}\left(P_{n}\right)$. Let $\mathrm{G}$ be the graph $\operatorname{Spl}_{m}\left(P_{n}\right)$ described as indicated in Figure 7

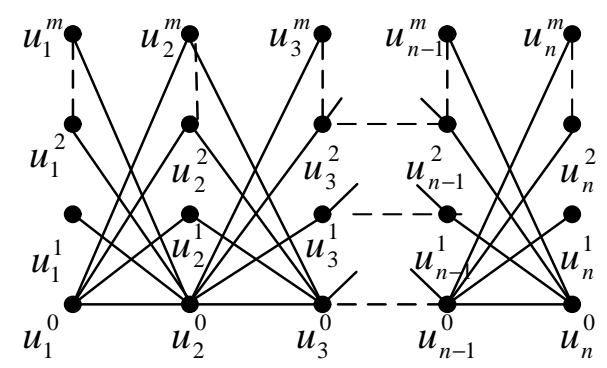

Figure 7

Then $|V(G)|=n(m+1)$ and $q=|E(G)|=(n-1)(2 m+1)$. We define

$$
f: V(G) \rightarrow\{0,1,2, \ldots, 2(n-1)(2 m+1)-1\}
$$

as follows: 
International journal on applications of graph theory in wireless ad hoc networks and sensor networks (GRAPH-HOC) Vol.5, No.2, June 2013

$$
\begin{aligned}
& f\left(u_{i}^{0}\right)= \begin{cases}2 q-i, & i=1,3,5, \ldots, n \text { or } n-1, \\
i-2 & i=2,4,6, \ldots, n-1 \text { or } n .\end{cases} \\
& f\left(u_{i}^{j}\right)= \begin{cases}2 q-i-4(n-1) j, & i=1,3,5, \ldots, n \text { or } n-1, \quad 1 \leq j \leq m, \\
2(n-1)(2 j-1)+i-2, & i=2,4,6, \ldots, n-1 \text { or } n, \quad 1 \leq j \leq m .\end{cases}
\end{aligned}
$$

The above defined function $f$ provides odd graceful labeling for the graph $\operatorname{Spl}_{m}\left(P_{n}\right)$. Hence $\mathrm{Spl}_{m}\left(P_{n}\right)$ is an odd graceful graph.

Example 3.2. Odd graceful labeling of the graph $\operatorname{Spl}_{4}\left(P_{7}\right)$ is shown in Figure 8.

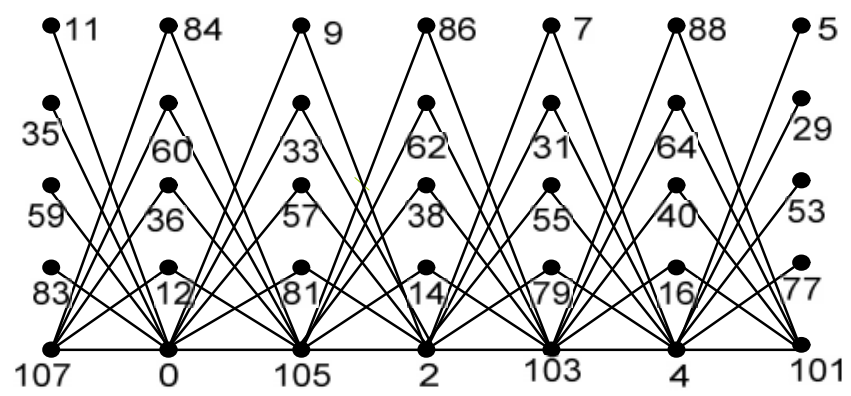

Figure 8: The graph $\operatorname{Spl}_{4}\left(P_{7}\right)$ with its odd graceful labeling.

Theorem 3.3. The graph $\operatorname{Spl}_{m}\left(K_{l, n}\right)$ is odd graceful.

Proof. Let $u_{1}, u_{2}, u_{3}, \ldots, u_{n}$ be the pendant vertices and $u_{0}$ be the centre of $K_{l, n}$, and $u_{0}^{j}, u_{1}^{j}, u_{2}^{j}, \ldots, u_{n}^{j}, 1 \leq j \leq m$ are the added vertices corresponding to $u_{0}, u_{1}, u_{2}, u_{3}, \ldots, u_{n}$ to obtain $\operatorname{Spl}_{m}\left(K_{l, n}\right)$. Let $G$ be the graph $\operatorname{Spl}_{m}\left(K_{l, n}\right)$. Then $|V(G)|=(n+1)(m+1)$ and $q=|E(G)|=$ $n(2 m+1)$. We define the vertex labeling function:

$f: V(G) \rightarrow\{0,1,2, \ldots, 2 n(2 m+1)-1\}$ as follows:

$f\left(u_{0}\right)=2 q-1$,

$$
f\left(u_{i}\right)=2(i-1), \quad 1 \leq i \leq n,
$$

$f\left(u_{0}^{j}\right)=(2 q-1)-2 n j, \quad 1 \leq j \leq m$,

$f\left(u_{i}^{j}\right)=2 n(m+j)+2(i-1), \quad 1 \leq i \leq n, 1 \leq j \leq m$.

In view of the above defined labeling pattern the graph under consideration admits odd graceful labeling. Hence $\operatorname{Spl}_{m}\left(K_{l, n}\right)$ is an odd graceful graph.

Example 3.4. An odd graceful labeling of the graph $\operatorname{Spl}_{2}\left(K_{1,4}\right)$ is shown in Figure 9 . 
International journal on applications of graph theory in wireless ad hoc networks and sensor networks (GRAPH-HOC) Vol.5, No.2, June 2013

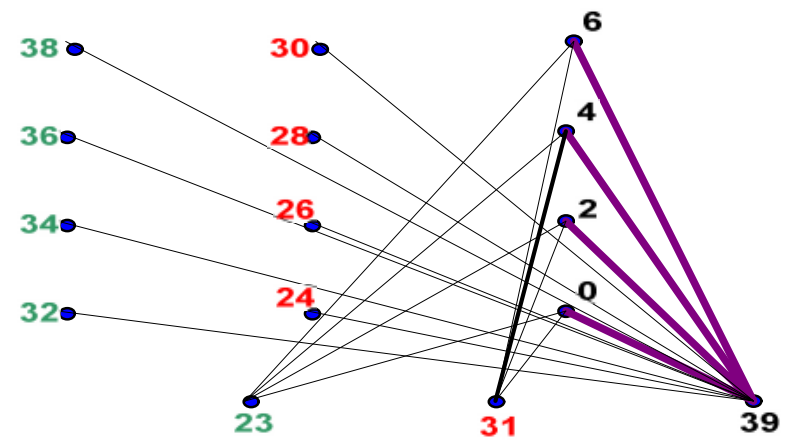

Figure 9: The graph $\operatorname{spl}_{2}\left(K_{1,4}\right)$ with its odd graceful labeling.

Theorem 3.5.

The graphs $\operatorname{Spl}_{m}\left(P_{n} \oplus \overline{K_{2}}\right), m, n \geq 2$ are odd graceful.

Proof. Let $u_{1}, u_{2}, u_{3}, \ldots, u_{n} ; v_{1}, v_{2}, v_{3}, \ldots, v_{n}$ be the vertices of the graph $P_{n} \oplus \overline{K_{2}}$ and suppose $u_{1}^{j}, u_{2}^{j}, u_{3}^{j}, \ldots, u_{n}^{j}, \quad 1 \leq j \leq m$ be the $\mathrm{j}^{\text {th }}$ vertices corresponding to $u_{1}, u_{2}, u_{3}, \ldots, u_{n}$ and $v_{1}^{j}, v_{2}^{j}, v_{3}^{j}, \ldots, v_{n}^{j}, 1 \leq j \leq m$ be the $\mathrm{j}^{\text {th }}$ vertices corresponding to $v_{1}, v_{2}, v_{3}, \ldots, v_{n}$ which are added to obtain $\operatorname{Spl}_{m}\left(P_{n} \oplus \overline{K_{2}}\right)$. The graph $\operatorname{Spl}_{m}\left(P_{n} \oplus \overline{K_{2}}\right)$ is described as indicated in Figure 10.

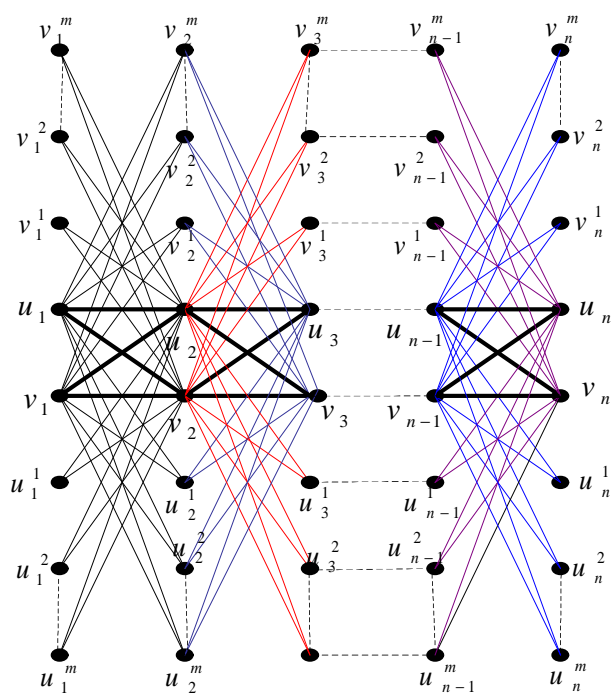

Figure 10

Then the number of edges of the graph $\operatorname{Spl}_{m}\left(P_{n} \oplus \overline{K_{2}}\right)=4(2 m+1)(n-1)$. 
International journal on applications of graph theory in wireless ad hoc networks and sensor networks (GRAPH-HOC) Vol.5, No.2, June 2013

We define:

$f: V\left(\operatorname{Spl}_{m}\left(P_{n} \oplus \overline{K_{2}}\right)\right) \rightarrow\{0,1,2, \ldots 8(2 m+1)(n-1)-1\}$.

First, we consider the labeling for the graph $P_{n} \oplus \overline{K_{2}}$ as follows:

$$
\begin{aligned}
f\left(u_{i}\right)= \begin{cases}2[q-2(2 m+1) i+2 m]-1, & i=1,3,5, \ldots n \text { or } n-1 \\
4[(2 m+1) i-4 m]-6, & i=2,4,6, \ldots n-1 \text { or } n .\end{cases} \\
f\left(v_{i}\right)= \begin{cases}2[q-2(2 m+1) i+2 m]+3, & i=1,3,5, . . n \text { or } n-1 \\
4(2 m+1)(i-2), & i=2,4,6, . . n-1 \text { or } n .\end{cases}
\end{aligned}
$$

For labeling the added vertices $u_{i}^{j}, v_{i}^{j}, 1 \leq i \leq n, 1 \leq j \leq m$ we consider the following two cases:

Case $(i):$ if $i$ is odd, $1 \leq i \leq n$ we have the following labeling, for each $1 \leq j \leq m$

$$
\begin{gathered}
f\left(u_{i}^{j}\right)=2[q-2(2 m+1) i-2 j+4 m]+7, \\
f\left(v_{i}^{j}\right)=2[q-2(2 m+1) i-2 j+2 m]-1
\end{gathered}
$$

Case(ii): if $i$ even, $2 \leq i \leq n$ and $1 \leq j \leq m$ we have the following labeling:

$$
f\left(u_{i}^{j}\right)=\left\{\begin{array}{lc}
4[(2 m+1) i+j-3 m-1], & j=1,3,5, \ldots m \text { or } m-1 \\
4[(2 m+1) i+j-3 m]-6, & j=2,4,6, \ldots m-1 \text { or } m .
\end{array}\right.
$$

Now we label the remaining vertices $v_{i}^{j}$,

if $i$ even, $2 \leq i \leq n$ and $m \equiv 1(\bmod 2), 1 \leq j \leq m$ we have the following labeling:

$$
f\left(v_{i}^{j}\right)= \begin{cases}4[(2 m+1) i+j-2 m]-6, & j=1,3,5, . . m \\ 4[(2 m+1) i+j-2 m-1], & j=2,4,6, . . m-1\end{cases}
$$


International journal on applications of graph theory in wireless ad hoc networks and sensor networks (GRAPH-HOC) Vol.5, No.2, June 2013

if $i$ even, $2 \leq i \leq n$ and $m \equiv 0(\bmod 2), 1 \leq j \leq m$ we have the following labeling:

$$
f\left(v_{i}^{j}\right)= \begin{cases}4[(2 m+1) i+j-2 m-1], & j=1,3,5, . . m-1 \\ 4[(2 m+1) i+j-2 m]-6, & j=2,4,6, . . m .\end{cases}
$$

In accordance with the above labeling pattern the graph under consideration admits odd graceful labeling. Hence $\operatorname{Spl}_{m}\left(P_{n} \oplus \overline{K_{2}}\right)$ is an odd graceful graph.

Example 3.6. Odd graceful labelings of graphs $\operatorname{Spl}_{2}\left(P_{4} \oplus \overline{K_{2}}\right)$ and $\operatorname{Spl}_{3}\left(P_{4} \oplus \overline{K_{2}}\right)$ are shown in Figure (11a) and Figure (11b) respectively.

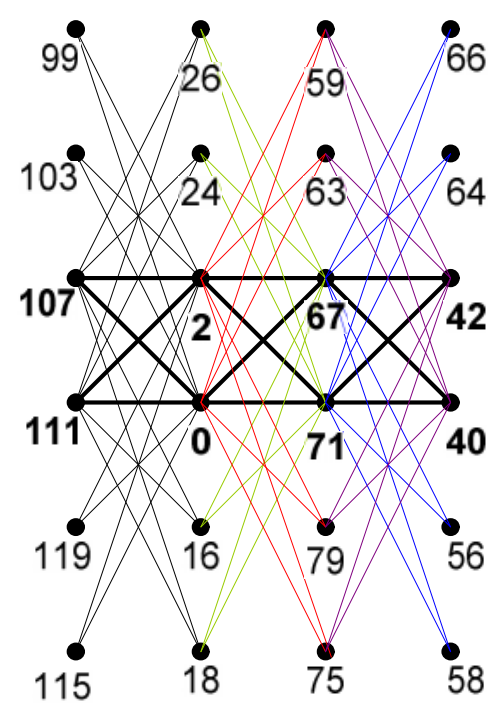

Figure (11a) $m \equiv 0(\bmod 2)$,

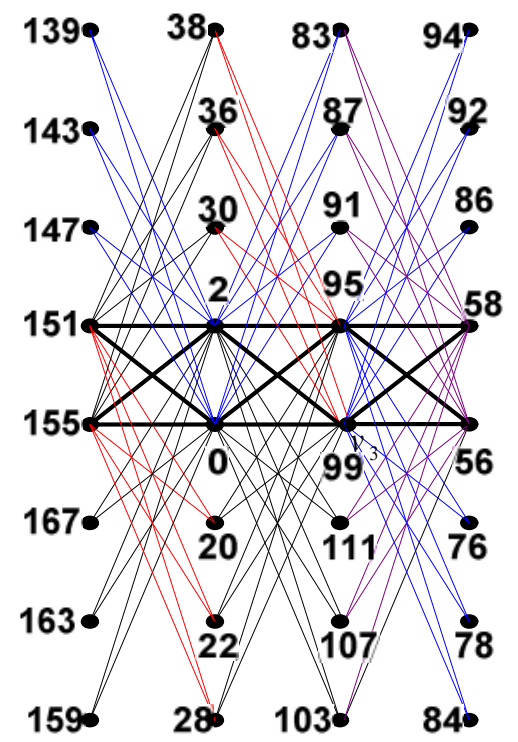

Figure $(11 \mathrm{~b}), m \equiv 1(\bmod 2)$

Figure (11a), Figure (11b): The graphs $\operatorname{spl}_{2}\left(P_{4} \oplus \overline{K_{2}}\right)$ and $\operatorname{Spl}_{3}\left(P_{4} \oplus \overline{K_{2}}\right)$ with their odd graceful labelings respectively.

\section{Remark 3.7.}

In Theorem 3.1, 3.3, 3.5 if we take $m=1$ we obtain the known splitting graphs (path, star and $P_{n}$ $\oplus \overline{K_{2}}$; respectively). These special cases of our results are coincided with the results which had been obtained in the articles (Sekar [10], Vaidya and Shah [9], Seoud and Abdel-Aal.[7] ; respectively). 
International journal on applications of graph theory in wireless ad hoc networks and sensor networks (GRAPH-HOC) Vol.5, No.2, June 2013

\section{CONCLUSION}

Since labeled graphs serve as practically useful models for wide-ranging applications such as communications network, circuit design, coding theory, radar, astronomy, X-ray and crystallography, it is desired to have generalized results or results for a whole class, if possible. In this work we contribute two new graph operations and several new families of odd graceful graphs are obtained. To investigate similar results for other graph families and in the context of different labeling techniques is open area of research.

\section{REFERENCES}

[1] G. S. Bloom and S. W. Golomb, (1977) "Applications of numbered undirected graphs", Proc. IEEE, Vol. 65, pp. 562-570.

[2] A. Rosa, (1967) On certain valuations of the vertices of a graph, in Theory of Graphs, International Symposium, Rome, July 1966, Gordon and Breach, NewYork and Dunod, Paris, pp. 349-355.

[3] S.W. Golomb, (1972) "How to number a graph, in Graph Theory and Computing", R.C. Read, ed.,Academic Press, NewYork, pp. 23-37.

[4] J. A. Gallian, (2012) A Dynamic Survey of Graph Labeling, Electronic J. Combin. Fiftteenth edition.

[5] F. Harary, (1969) GpaphTheory, Addison-Wesley, Reading MA.

[6] R.B. Gnanajothi, (1991) Topics in graph theory, Ph.D. thesis, Madurai Kamaraj University, India.

[7] M.A. Seoud and M.E. Abdel-Aal, (2013) "On odd graceful graphs", Ars Combin., Vol. 108, pp.161185.

[8] S.K. Vaidy and B. Lekha, (2010) "New Families of Odd Graceful Graphs", Int. J. Open Problems Compt. Math., Vol. 3, No. 5, pp. 166-171.

[9] S.K. Vaidy and B. Lekha, (2010) "Odd Graceful Labeling of Some New Graphs", Modern Applied Science Vol. 4, No. 10, pp. 65-70.

[10] C.Sekar, (2002) Studies in Graph Theory, Ph.D. Thesis, Madurai Kamaraj University.

[11] M. A. Seoud and E. A. Elsahawi, (2008) On variations of graceful labelings, Ars Combinatoria, Vol. 87, pp. 127-138.

\section{AUTHOR}

Mohamed Elsayed Abdel-Aal received the B.Sc. (Mathematics) the M.Sc.(Pure Mathematics-Abstract Algebra) degree from Benha University, Benha, Egypt in 1999, 2005 respectively. Also, he received Ph.D. (Pure Mathematics) degree from Faculty of Mathematics, Tajik National University, Tajikistan, in 2011. He is a University lecturer of Pure Mathematics with the Ben ha University, Faculty of Science, Department of Pure Mathematics. His current research is Ordinary -partial differential equations, Graph Theory and Abstract Algebra.

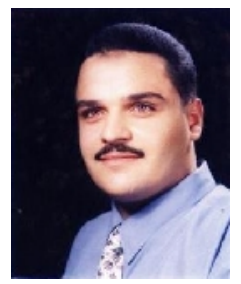

